\title{
Impaired baseline regional cerebral perfusion in patients referred for coronary artery bypass
}

Robert Moraca, MD, ${ }^{b}$ Eugene Lin, MD, ${ }^{c}$ James H. Holmes IV, MD, ${ }^{d}$ David Fordyce, PhD, ${ }^{a}$ William Campbell, PA-C, ${ }^{a}$ Mary Ditkoff, PA-C, ${ }^{a}$ Mark Hill, MD, ${ }^{a}$ Steven Guyton, MD, ${ }^{a}$ Daniel Paull, MD, and R. Alan Hall, MD ${ }^{a}$

Background: Cognitive dysfunction and cerebral vascular accidents remain some of the most devastating problems related to cardiac surgery. Despite the major advances in perioperative care and operative technique in coronary artery bypass, this cohort of patients appears to have poor cerebral physiologic reserve. The aim of this study was to describe regional cerebral perfusion of patients with coronary artery disease referred for coronary artery bypass grafting.

Methods: Eighty-two consecutive patients with coronary artery disease referred for coronary artery bypass grafting were enrolled after providing informed consent in an institutional review board-approved study. Patients with prior cerebral vascular accident, transient ischemic attacks, head trauma, or other neurologic afflictions were excluded from the study. We prospectively measured preoperative regional cerebral perfusion using single photon emission computed tomography (SPECT) imaging of 12 regions. Patients were determined to have an abnormal SPECT if regional cerebral perfusion was less than 2 standard deviations below the mean of age-matched controls.

Results: The mean age was 67.5 (range, 34-89) years. The study group comprised $22 \%$ women and $78 \%$ men with known risk factors for atherosclerosis: current tobacco use (30\%), hypertension (69\%), and diabetes (27\%). Seventy-five percent of the SPECT scans demonstrated abnormal regional cerebral perfusions, which were associated with older age $(P<.008)$, current tobacco use $(P<.005)$, and diabetes mellitus $(P<.005)$. The incidence of postoperative cerebral vascular accident was $5 \%$ and only occurred in patients with abnormal regional cerebral perfusion.

From the Departments of Cardiothoracic Surgery, ${ }^{\mathrm{a}}$ General Surgery, ${ }^{\mathrm{b}}$ and Radiology, ${ }^{\mathrm{c}}$ Virginia Mason Medical Center, Seattle, Wash., and the Burn Service, ${ }^{\mathrm{d}}$ Wake Forest University, Winston-Salem, N.C.

Read at the Thirty-first Annual Meeting of The Western Thoracic Surgical Association, Victoria, BC, Canada, June 22-25, 2005.

Received for publication July 7, 2005; revisions received Oct 10, 2005; accepted for publication Oct 20, 2005.

Address for reprints: Robert J. Moraca, MD, Virginia Mason Medical Center, 5405 Baker Ave NW, Seattle, WA, 98107 (E-mail: robert. moraca@vmmc.org).

J Thorac Cardiovasc Surg 2006;131:540-6

$0022-5223 / \$ 32.00$

Copyright $\odot 2006$ by The American Association for Thoracic Surgery

doi:10.1016/j.jtcvs.2005.10.046
Conclusion: Seventy-five percent of patients undergoing coronary bypass grafting have a significant impairment in regional cerebral perfusion compared with published age-matched controls, which may contribute to their proclivity for cerebral complications.

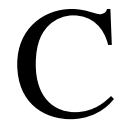
erebral complications can be some of the most devastating problems after cardiac surgery. Etiologies of postoperative cerebral perturbations have been linked to cardiopulmonary bypass, microembolic and macroembolic events, hypoperfusion, hyperthermia, and the systemic inflammatory response. Although efforts to manipulate intraoperative variables have resulted in a reduction in cerebral complications; the incidence of postoperative cerebral vascular accidents (CVA) and cognitive dysfunction is still reported to be as high as $1 \%$ to $6 \%$ and up to $80 \%$ respectively. ${ }^{1-4}$ This cohort of patients with significant coronary artery disease (CAD) may be at higher risk for cerebral complications attributable to cerebral microvascular and macrovasculature disease, resulting in a limited cerebral physiologic reserve. Several studies have documented "silent" cerebral infarcts on computed tomography (CT) and magnetic resonance imaging (MRI) in patients with 


\author{
Abbreviations and Acronyms \\ CVA = cerebral vascular accidents \\ $\mathrm{CT}=$ computed tomography \\ $\mathrm{CABG}=$ coronary artery bypass grafting \\ $\mathrm{CAD}=$ coronary artery disease \\ $\mathrm{CVA}=$ cerebral vascular accident \\ LVEF = left ventricular ejection fraction \\ LOS $=$ length of stay \\ MRI = magnetic resonance imaging \\ $\mathrm{rCP}=$ regional cerebral perfusion \\ SPECT $=$ single photon emission computed \\ tomography \\ ${ }^{99 \mathrm{~m}} \mathrm{Tc}=$ technetium-99m \\ TIA $=$ transient ischemic attack
}

CAD. ${ }^{5-8}$ However, these modalities may underestimate the degree of cortical impairment by not reflecting areas of chronic ischemia or watershed areas maintained only through dysfunctional autoregulation with maximal vasodilation. ${ }^{9,10}$

Single photon emission tomography (SPECT) uses technetium-based radioisotopes attached to ligands, which are actively bound to cerebral endothelial membranes, providing highly sensitive information regarding metabolism and regional cerebral blood flow patterns. ${ }^{11-12}$ In addition, SPECT may illustrate areas of diaschisis, cortical hypofunction in an area linked to ischemic cortex, and deafferentation. The aim of this study was to describe regional cerebral perfusion $(\mathrm{rCP})$ in patients with $\mathrm{CAD}$ referred for coronary artery bypass grafting (CABG) and compare it with published age-matched controls. ${ }^{13}$

\section{Methods}

Eighty-two neurologically normal patients with CAD referred for first-time CABG were enrolled prospectively and underwent SPECT imaging between October 2000 and May 2001 in a study approved by theVirginia Mason Medical Center Institutional Review Board. Staff surgeons and cardiothoracic physician assistants preoperatively evaluated each patient by history and physical examination. Any patient with prior CVA, transient ischemic attacks (TIA), head trauma, and clinical evidence of carotid artery disease and other neurologic afflictions were excluded from the study. Patients with chronic renal insufficiency $(\mathrm{Cr} \geq 1.6)$ also were excluded because of possible perturbations in the pharmacokinetics of the radioisotope. Demographic and clinical data were collected prospectively and recorded into the research database at the time of cardiothoracic consultation.

\section{SPECT Brain Perfusion Scanning}

All patients were imaged supine with an ADAC Dual Head Genesys Gamma Camera linked to a Pegasys computer system (ADAC Laboratories, Milpitas, CA). The SPECT tracer employed, technetium-99m $\left({ }^{99 \mathrm{~m}} \mathrm{Tc}\right)$ Bicisate (Neurolite; DuPont Pharma, Wilmington, Del) is a technetium-based Federal Drug Administrationapproved radioisotope and has been shown comparable with xenon
133 and ${ }^{99 \mathrm{~m}} \mathrm{Tc}$ HMPAO in cerebral SPECT studies. ${ }^{14}$ Acceptability within subject reproducibility of technetium SPECT scans has been demonstrated, supporting its use in same subject repetitive scan studies. ${ }^{12}{ }^{99 \mathrm{~m}} \mathrm{Tc}$-bicisate forms a stable lipophilic complex, crosses the blood-brain barrier, and is passively diffused across intact cell membranes. The radioisotope is stable within the brain, can be scanned up to 6 hours after injection, and yields a snapshot image of cerebral perfusion at the time the isotope was administered.

All patients were injected with ${ }^{99 \mathrm{~m}} \mathrm{Tc}$-bicisate preoperatively and scanned 3 hours after radioisotope injection. A Functional Brain Threshold Analysis program (ADAC Laboratories, Milpitas, Calif) was used by a single radiologist for scan analysis. This software consists of a visual quantitation program based on thresholding to the mean pixel value in the visually normal cerebellar hemispheres. The regions analyzed were the inferior frontal, superior frontal, posterior temporal, lateral temporal, anterior parietal, and posterior parietal. The basal ganglia and thalamus were excluded because of their relative diminutive size. The region-tocerebellum ratios were evaluated in comparison with a previously published database of region-to-cerebellum ratios in healthy adult volunteers, which defined the normal region-to-cerebellum ratio, the standard deviation, and the expected age-related decline for each region. ${ }^{13}$ Age-related perfusion decreases of $1 \%$ to $2 \%$ per decade are expected in the superior frontal $(-1.4 \%)$, anterior parietal $(-1.3 \%)$, posterior parietal $(-1.3 \%)$, and basal ganglia $(-1.6 \%)$ regions. The expected normal region-to-cerebellum ratios in each region were adjusted for age according to these previously published values. For each study, the region-to-cerebellum ratios in each region were compared with the expected normal age-adjusted ratio and standard deviation. Regions containing an area of decreased perfusion with a ratio of 2 or more standard deviations below the expected normal ratio were considered to have an $\mathrm{rCP}$ defect. SPECT scans were scored on the basis of the total number of rCP defects: normal ( 0 defects), mild (1-2 defects), moderate (3-4 defects), and severe ( $>4$ defects).

\section{Cardiopulmonary Bypass and Anesthesia}

Cardiopulmonary bypass was used on all coronary revascularizations. Induction was accomplished with midazolam $(0.2-0.3 \mathrm{mg} /$ $\mathrm{kg}$ ). Fentanyl was used as indicated, subject to the judgment of the attending anesthesiologist. Intubation was facilitated with pancuronium $(10 \mathrm{~mL})$. Bolus or constant infusion of midazolam and fentanyl combinations was used as indicated, with supplementary pancuronium to provide adequate muscle relaxation. Isoflurane up to a maximum of $0.5 \%$ (end-tidal) was used to supplement anesthesia, as needed, throughout the case. Cardiopulmonary bypass was performed, following standard institutional protocol, with systemic cooling to $32^{\circ} \mathrm{C}$. Perfusion pressures were maintained between 45 and $60 \mathrm{~mm} \mathrm{Hg}$ with vasopressor (Neo-Synephrine) or vasodilator (nitroglycerin) administered when necessary. Systemic flow rates were maintained between 2.0 and $2.4 \mathrm{~L} \cdot \mathrm{min}^{-1} \cdot \mathrm{m}^{-2}$ and $\mathrm{pH}$ maintained by the alpha-stat method.

\section{Statistical Analysis}

Independent sample, 2-tailed Student $t$ tests, and chi-square were employed on demographic, surgical, SPECT, and outcome data 
TABLE 1. Study population demographics ( $N=82$ )

\begin{tabular}{lc}
\hline Demographics & \\
Age (mean [range]) (y) & $67.5(34-89)$ \\
Gender (men) & $78 \%$ \\
Education (mean [range]) (y) & $14.4(0-20)$ \\
LVEF & $56.9 \%$ \\
Hypertension & $68 \%$ \\
Diabetes mellitus & $28 \%$ \\
Operation & \\
CABG & $82 \%$ \\
CABG + valve & $18 \%$ \\
\hline
\end{tabular}

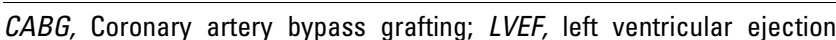
fraction.

using a statistical software package (StatView Software, SAS Institute; Cary, NC).

\section{Results}

Eighty-two neurologically normal patients were enrolled in the study. Eighteen percent $(n=15)$ were referred for CABG and valve replacement and $82 \%(n=67)$ for CABG alone. The study population comprised $78 \%$ men, and the mean age was 67.5 years (range, 34-89 years). Specific demographic risk factors for atherosclerosis included hyper- tension $68 \%$, diabetes mellitus $28 \%$, and tobacco use $33 \%$ (defined as any patient with a $>30$ pack-year history of smoking or a current smoker with a $>10$ pack-year history of smoking). The average education of the study group was 14.4 years (range, $0-20$ years; Table 1 ).

The $\mathrm{rCP}$ in 12 areas of the brain were normal in $25 \%$ $(n=20)$ of patients and abnormal in $75 \%(n=62)$ of patients. The severity of rCP defects in the abnormal group ranged from mild (28\%), moderate (24\%), and severe (23\%; Figure 1). The mean number of rCP defects was 3.6 (range, 0-10; Figure 2). The posterior temporal lobe, inferior frontal, superior frontal, with equal left and right distribution, were affected in $>50 \%$ of patients with abnormal SPECT. The anterior and posterior parietal lobe had the lowest incidence of rCP deficits (Table 2). Twenty-five percent of the patients had rCP deficits involving $75 \%$ to $100 \%$ of their entire frontal lobe.

Abnormal rCP correlated significantly with older age (69.5 vs $62.4, P \leq .008)$, diabetes mellitus (9\% vs 33\%, $P<$ $.05)$, tobacco use $(16 \%$ vs $39 \%, P<.05)$, and CABG totaling 4 or more ( $15 \%$ vs $48 \%, P \leq .02$ ). There were no correlations with hypertension, education, gender, or left ventricular ejection fraction (LVEF) (Table 3). Patients who underwent $\mathrm{CABG}$ alone were significantly more likely to
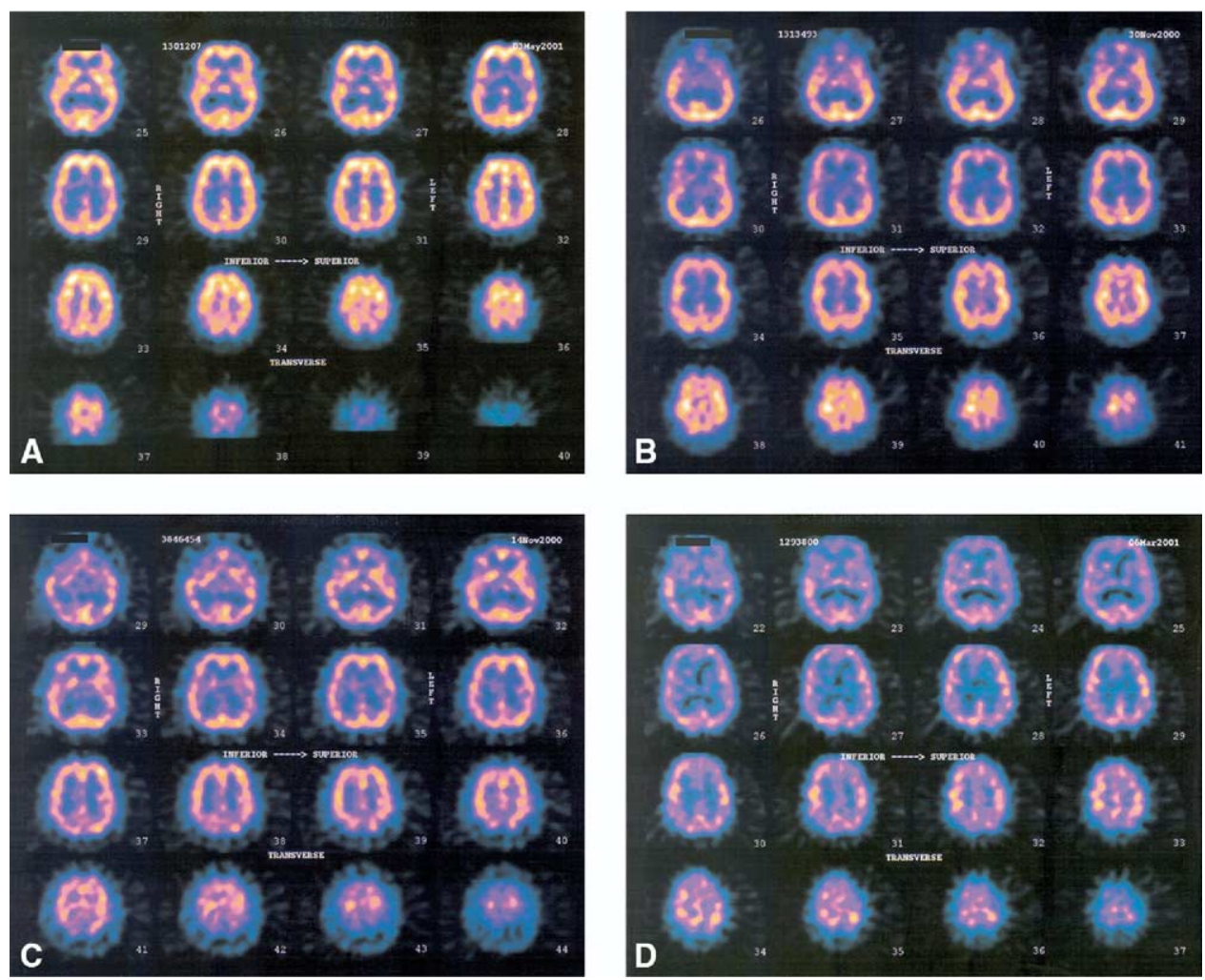

Figure 1. Regional cerebral perfusion by SPECT. SPECT images above are examples and distribution of the four classifications. Top left, normal (0 rCP defects) $25 \%$. Top right, mild (1-2 rCP defects) $28 \%$. Bottom left, moderate (3-4 rCP defects) 24\%. Bottom right, severe (>4 rCP defects) $23 \%$. 


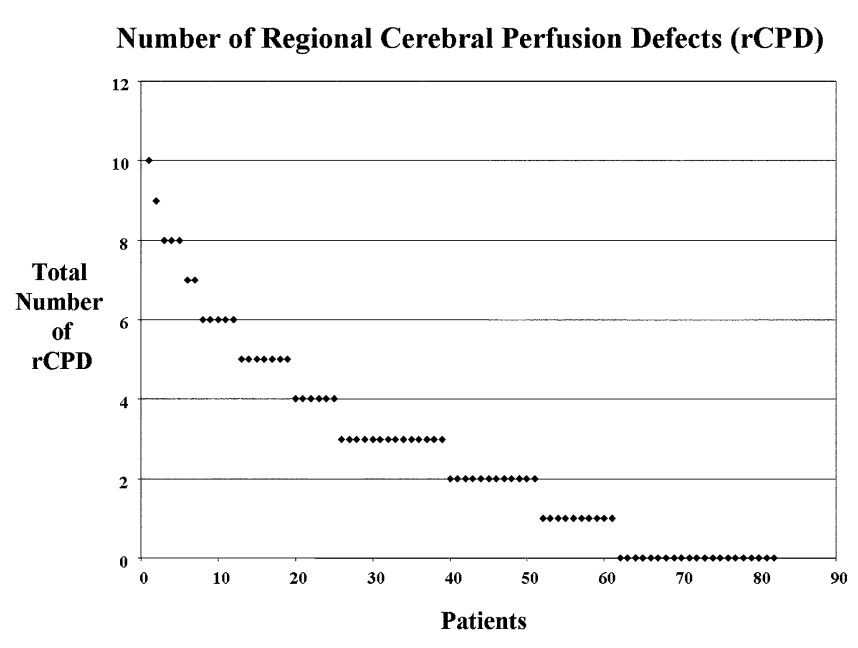

Figure 2. Number of regional cerebral perfusion defects (rCPD).

have abnormal rCP patients than those who underwent CABG/valve $(53 \%$ vs $79 \%, P \leq .01)$.

The mean length of stay (LOS), intensive care unit LOS, postoperative cerebral vascular accidents (CVA), and 30day mortality are reported in Table 4. Three patients had documented postoperative CVA, and 2 perioperative deaths occurred, 1 in a patient with a postoperative stroke.

\section{Discussion}

In 1998, our group found that abnormal preoperative rCP on SPECT was a strong indicator for postsurgical decline in neuropsychologic testing. ${ }^{15}$ In addition, we were impressed at the overall number of preoperative abnormal SPECTs in many of the neurologically normal patients, yet we had no control group to define this as a normal age-related process or a process associated with widespread atherosclerosis. In 2000, Tanaka and associates ${ }^{13}$ published the first normative database for rCP in healthy adults. This database enabled us to rigorously study baseline $\mathrm{rCP}$ values in patients referred for $\mathrm{CABG}$ and compare them with published values for healthy, age-matched controls.

Previous studies of "normal" patients with CAD have demonstrated preoperative cognitive defects by neuropsychologic testing. Rankin and associates ${ }^{16}$ in 2003 adminis-

TABLE 2. Regional cerebral perfusion defects on SPECT

\begin{tabular}{lc}
\hline Region & Distribution of perfusion deficits \\
\hline Inferior frontal & $35 / 62(56 \%)$ \\
Superior frontal & $32 / 62(52 \%)$ \\
Posterior temporal & $37 / 62(60 \%)$ \\
Lateral temporal & $23 / 62(37 \%)$ \\
Anterior parietal & $17 / 62(27 \%)$ \\
Posterior parietal & $15 / 62(24 \%)$ \\
\hline
\end{tabular}

TABLE 3. Demographic and clinical variables associated with SPECT

\begin{tabular}{lccc}
\hline Variable & $\begin{array}{c}\text { Normal } \\
\text { SPECT } \\
(\mathbf{n}=\mathbf{2 0})\end{array}$ & $\begin{array}{c}\text { Abnormal } \\
\text { SPECT } \\
(\mathbf{n}=\mathbf{6 2})\end{array}$ & $\boldsymbol{P}$ value \\
\hline Gender (\% male) & $85 \%$ & $75 \%$ & $\mathrm{NS}$ \\
Education (mean \pm SD) (y) & $14.5 \pm 2.8$ & $14.7 \pm 3.4$ & $\mathrm{NS}$ \\
Age (mean \pm SD) (y) & $62.4 \pm 9.8$ & $69.8 \pm 11.8$ & $P<=.008$ \\
Hypertension & $64 \%$ & $69 \%$ & $\mathrm{NS}$ \\
Current tobacco use & $16 \%$ & $39 \%$ & $P \leq .05$ \\
Diabetes mellitus & $9 \%$ & $33 \%$ & $P \leq .05$ \\
LVEF (mean \pm SD) (\%) & $59.3 \pm 7.4$ & $56.3 \pm 13.9$ & $\mathrm{NS}$ \\
CABG $\times \geq 4$ & $15 \%$ & $48 \%$ & $P \leq .02$ \\
\hline
\end{tabular}

tered preoperative and postoperative intensive neuropsychologic testing to $43 \mathrm{CABG}$ patients randomized to either off-pump or cardiopulmonary bypass revascularization. Significant impairments were demonstrated in multiple domains, particularly in the areas of verbal memory and psychomotor skills in patients preoperatively, which were unchanged postoperatively at 2.5 months in either group. Thus, patients may come to surgery with significant cognitive impairment, and, perhaps, cardiopulmonary bypass does not play a major role in postoperative cognitive decline. Is cognitive decline a reflection of a preexisting diffuse vascular process common in all patients with CAD? A prospective study of neurologic and neuropsychologic complications after major vascular surgery or CABG found that $61 \%$ and $79 \%$, respectively, developed early neurologic and/or neuropsychologic abnormalities. ${ }^{17}$ In addition, the Stent or Surgery (SoS) Trial's failure to find a statistical difference in neuropsychologic outcomes in 2 randomized revascularization groups, $\mathrm{CABG}$ or percutaneous coronary, at 6 or 12 months implies that patients with CAD are prone to cerebral perturbations regardless of revascularization technique. $^{18}$

In an attempt to determine the potential etiology of cognitive impairment in this group, several investigators ${ }^{5-7,17,19}$ have demonstrated subtle neurological abnormalities and MRI defects are present in patients with coronary atherosclerosis. However, many patients with normal preoperative

TABLE 4. Clinical outcomes associated with SPECT

\begin{tabular}{lccc}
\hline Clinical variables & $\begin{array}{c}\text { Normal SPECT } \\
(\mathbf{n}=\mathbf{2 0})\end{array}$ & $\begin{array}{c}\text { Abnormal SPECT } \\
(\mathbf{n}=\mathbf{6 2})\end{array}$ & $\begin{array}{c}\boldsymbol{P} \\
\text { value }\end{array}$ \\
\hline LOS (mean \pm SD) (d) & $5.0 \pm 2.3$ & $5.9 \pm 4.5$ & $\mathrm{NS}$ \\
Discharge to SNF/RHU & $10 \%$ & $23 \%$ & $\mathrm{NS}$ \\
Postoperative CVA & $0 \%$ & $5 \%$ & $\mathrm{NS}$ \\
Death & $0 \%$ & $3 \%$ & $\mathrm{NS}$
\end{tabular}

CVA, Cerebral vascular accident; $L O S$, length of stay; $R H U$, rehabilitation; $S N F$, short-term nursing facility. 
and postoperative head MRI and CT also will have postoperative cognitive defects. Thus, we employed a more powerful instrument, SPECT, to examine rCP. SPECT has the advantage of measuring $\mathrm{rCP}$ using the radioisotope ${ }^{99 \mathrm{~m}} \mathrm{Tc}-$ bicisate, which forms a stable lipophilic complex, crosses the blood-brain barrier, and captures a snapshot in $\mathrm{rCP}$, thus revealing areas of chronic ischemia, watershed areas maintained only through dysfunctional autoregulation. The ischemia causes regional vasodilation to maintain direct and/or collateral perfusion to ischemic or infarcted cerebral tissue. Perhaps poor cerebral perfusion results in a diminished cerebral physiologic reserve, limiting the ability to tolerate the variety of insults of hypoperfusion, inflammation, and embolic phenomena, which may occur during surgery. Our study did not investigate directly whether an intact cerebral autoregulatory system was present by employing a vasodilator challenged test using inhaled carbon dioxide, acetazolamide, or adenosine. These tests would unmask further areas that may appear normal on our nonchallenged ${ }^{99 \mathrm{~m}} \mathrm{Tc}-$ bicisate study. We use the term "diminished cortical reserve" only to reflect overall impaired cortical tissue at baseline.

Eighty percent of the patients in our study were male, reflecting that the published predominance in incidence, diagnosis, and treatment of CAD has been well documented in the United States and Europe. ${ }^{20}$ In this group we found that $75 \%$ of neurologically normal patients referred for CABG had areas of abnormal rCP, compared with published age-matched controls. Our SPECT scoring system was developed to critically identify regions of the brain that rCP indicated was significantly impaired. Even though many of our study subjects had areas that were greater than 1 to 1.8 standard deviations outside the mean, we chose to define an $\mathrm{rCP}$ abnormality with strict criteria: any region with more than 2 standard deviations outside the mean and adjusted for normal, age-matched, expected decline. The basal ganglia and thalamus were excluded, and the other 12 regions were considered equal. Thus, these criteria may ensure that only the most cognitively important and significantly impaired areas would be included. Our scoring system was developed by classifying cortical disease as a percentage of the overall cortex: normal $(0 \%)$, mild $(8 \%$ $16 \%)$, moderate $(25 \%-33 \%)$, and severe $(>41 \%)$.

Forty-eight percent of the patients had 3 or more rCP defects, estimated to be roughly $25 \%$ of their cortex. Not surprisingly, age, diabetes mellitus, and tobacco use all were associated with rCP defects. Older age is a well-described association with cognitive decline and perioperative stroke. ${ }^{21}$ Although patients with abnormal SPECT tended to be older, there are several notable exceptions within our group. The youngest 3 patients in our abnormal group (range, 43-54 years) all had 5 or more regional cortical blood flow abnormalities (grade, severe). Surprisingly, $41 \%$ of their studied cortical perfusion was abnormal, and yet at time of consultation they were neurologically normal. Although older age was associated with abnormal perfusion, it by no means implies that younger patients are likely to be spared.

Patients with rCP defects were more likely to have diabetes mellitus and use tobacco than were patients with normal SPECT. Atherosclerotic lesions developing in association with diabetes mellitus and tobacco use can occur as early as the second decade of life and progress into clinical disease over time. The mechanism of diabetic angiopathy is complex and still speculative; however, it is clearly a diffuse process not sparing the cerebral vasculature. Atherosclerosis of the coronary vessels usually translates into thoracic aorta and cerebral disease with subsequent increased macroembolization and microembolization, and $\mathrm{rCP}$ impairment, respectively. Reduced LVEF can contribute to hemodynamic instability, leading to poor cerebral perfusion, thrombosis, and possible CVA and/or other neurologic injuries. Although studies have found LVEF to be an important determinant of postoperative CVA, in our study it was not predictive of preoperative abnormal $\mathrm{rCP}^{21}$ Yet patients with abnormal rCP may be less tolerant of poor LVEF.

Patients who underwent $\mathrm{CABG}$ alone were significantly more likely to have abnormal rCP than those who underwent $\mathrm{CABG} /$ valve $(53 \%$ vs $79 \%, P \leq .01)$. Several groups have demonstrated higher postoperative cerebral complications after valve surgery, possibly linked to increased postoperative inflammation, operative embolic events, and preoperative chronic embolic events. However, more perfusion abnormalities were not demonstrated by small $\mathrm{CABG} / \mathrm{valve}$ cohort. Since the aim of our study was to determine the preoperative brain perfusion in patients with CAD, we chose to include this subgroup of patients with $\mathrm{CAD}$ and valve disease and to exclude patients with only valve disease.

Outcomes evaluated after coronary revascularization included LOS, discharge destination, postoperative CVA, and death. Although there was no statistical significance with abnormal rCP, patients with abnormal SPECT scans trended toward longer LOS (5.0 vs 5.9, not significant) and were more likely to be discharged to a short-term nursing facility or rehabilitation center (10\% vs. $23 \%$, not significant). In addition, there were 3 postoperative CVAs, all of which occurred in the patients with abnormal SPECT scans (grades I, II, and III). Left middle cerebral artery infarcts developed in 2 patients, and they were discharged to rehabilitation facilities. The third had a prolonged course in the intensive care unit for cardiopulmonary dysfunction and had a large, left, major coronary artery infarct with mass effect. The family elected to withdraw support. One other death attributable to severe cardiogenic shock occurred during revascularization.

Several limitations of our study should be noted. First, it is important to recognize that the SPECT studies were 
interpreted by a single radiologist employing a Functional Brain Threshold Analysis program, which objectively measures cerebral perfusion by calculating mean regional pixel values. However, the nonblinded radiologist, who is an author of the study, must select a location within the cerebral region to measure pixel values. Second, rigorous neuropsychologic testing and/or assessment by neurology staff was not conducted; rather, staff surgeons and physician assistants assessed each patient, which may have limited our ability to detect subtle defects. Finally, the small sample size of this observational cohort may limit its widespread applicability.

We have demonstrated that $75 \%$ of patients undergoing CABG have a significant impairment in baseline rCP, compared with published age-matched controls. Abnormal rCP may contribute to the proclivity for cerebral complications in this cohort of patients.

\section{References}

1. Carrascal Y, Guerrero AL, Maroto LC, et al. Neurological complications after cardiopulmonary bypass: an update. Eur Neurol. 1999; 41128-34.

2. Roach GW, Kanchuger M, Mora Mongano C, et al. Adverse cerebral outcomes after coronary bypass surgery. N Engl J Med. 1996;335: 1857-63.

3. Newman MF, Kirchner JL, Philips-Bute B, et al. Longitudinal assessment of neurocognitive function after coronary bypass surgery. $N \mathrm{Engl}$ J Med. 2001;344:395-402.

4. Murkin JM, Baird DL, Martzke JS, Adams SJ, Lok P. Long-term neurological and neuropsychological outcome 3 years after coronary bypass surgery [abstract] Anesth Analg. 1996;82:S328.

5. Modrego Pardo PJ, Labrador Fuster T, Torres Nuez J. Silent brain infarctions in patients with coronary heart diseases. A Spanish population survey. J Neurol. 1998;245:93-97.

6. Uekita K, Hasebe N, Funayama N, et al. Cervical and intracranial atherosclerosis and silent brain infarction in Japanese patients with coronary artery disease. Cerebrovasc Dis. 2003;1:61-8.

7. Restrepo L, Wityk RJ, Grega MA, et al. Diffusion and perfusion weighted magnetic resonance imaging of the brain before and after coronary artery bypass grafting surgery. Stroke. 2002;33:2909-15.

8. Kohn A. Magnetic resonance imaging registration and quantification of the brain and after coronary artery bypass graft surgery. Ann Thorac Surg. 2002;73:S363-5.

9. Powers WJ. Cerebral hemodynamics in ischemic cerebrovascular disease. Ann Neurol. 1991;29:231-40.

10. Yudd AP, Van Heertum RL, Masdeu JC. Interventions and functional brain imaging. Semin Nucl Med. 1991;21:153-8.

11. Devous MD. SPECT functional brain imaging. Technical considerations. J Neuroimaging. 1995;5(Suppl 1):S2-13.

12. Deutsch G, Mountz JM, Katholi CT, Liu HG, Harrell LE. Regional stability of cerebral blood flow measured by repeated technetium $99 \mathrm{~m}$ HMPAO SPECT: implications for the study of state-dependent change. J Nucl Med. 1997;38:6-13.

13. Tanaka F, Vines D, Tsuchida $T$, et al. Normal patterns on ${ }^{99 \mathrm{~m}} \mathrm{Tc}-\mathrm{ECD}$ brain SPECT scans in adults. J Nucl Med. 2000;41:1456-64.

14. Devous MD, Payne JK, Lowe JL. Comparison of 99m Tc-ECD to ${ }^{133} \mathrm{Xe}$ SPECT in normal controls and in patients with mild to moderate rCBF abnormalities. J Nucl Med. 1993;34:754-61.

15. Hall RA, Lee ME, Fordyce DI, et al. Cerebral SPECT imaging and neuropsychological testing in coronary artery bypass graft patients. Circulation. 1997;96(Suppl):727.

16. Rankin KP, Kochamba GS, Boone KB, et al. Presurgical cognitive deficits in patients receiving coronary artery bypass graft surgery. $J$ Int Neuropsychol Soc. 2003;9:913-24.
17. Shaw PJ, Bates D, Cartilage NEF, et al. Neurologic and neuropsychologic morbidity following major surgery: comparison of coronary artery bypass and peripheral vascular surgery. Stroke. 1987;14:700-17.

18. Wahrborg P, Booth JE, Clayton T, et al. Neuropsychological outcome after percutaneous coronary intervention or coronary artery bypass grafting: results from the Stent or Surgery (SoS) Trial. Circulation. 2004;110:3411-7.

19. Baird DL, Murkin JM, Lee DL. Neurologic findings in coronary artery bypass patients: perioperative or preexisting? J Cardiothorac Vasc Anesth. 1997;11:694-8.

20. Weisz D, Gusmano MK, Rodwin VG. Gender and the treatment of heart disease in older persons in the United States, France, and England: a comparative, population-based view of a clinical phenomenon. Gend Med. 2004;1:29-40.

21. Ancona GD, Saez de Ibarra JI, Baillot R, et al. Determinants of stroke after coronary artery bypass grafting. Eur J Cardio-Thorac Surg. 2003;24:552-6.

\section{Discussion}

Dr Mark Ratcliffe (San Francisco, Calif). To briefly summarize, Dr Moraca and colleagues have measured regional cerebral blood flow with single photon emission tomography, or SPECT, scan before $\mathrm{CABG}$ and $\mathrm{CABG}$ valve procedures and its association with stroke. The key finding is that $75 \%$ of $C A B G$ patients have at least 1 region of reduced regional cerebral blood flow. Age, current tobacco use, diabetes, and CABG greater than or equal to 4 were associated with reduced blood flow. There were 3 strokes in the cohort of patients with reduced regional cerebral blood flow and none in the normal SPECT scan group. However, the difference was unfortunately nonsignificant. Since the incidence of stroke and cognitive dysfunction may be as high as $6 \%$ and $50 \%$, respectively, the findings that many CABG patients enter the operating room with reduced regional cerebral blood flow is of significant interest.

I have three questions; the first two relate to mechanism and the third to your future plans.

Presumably the reduced regional cerebral blood flow is a manifestation of small vessel disease. Importantly, these areas of reduced regional cerebral blood flow may be vulnerable to cardiopulmonary bypass-mediated reductions in oxygen delivery. For instance, Gold has suggested that cerebral damage may occur when systemic pressure on bypass falls below the cerebral autoregulation threshold and Jonas and colleagues have suggested that cerebral damage may occur when the hematocrit is too low. My question is this: Was the surgeon aware of the preoperative SPECT scan results, and do you plan, in the future, to act differently when a preoperative $\mathrm{CABG}$ patient has an abnormal scan? For instance, was there any attempt to increase perfusion pressure and/or hematocrit on bypass or to do the case off-pump in the group with reduced regional blood flow?

Dr Moraca. Thank you for your questions. The surgeons were not aware of the SPECT results. The SPECT scans were interpreted at a separate time after the operation. Thus, they were not able to alter their intraoperative technique. The surgeons at our institution do maintain minimal perfusion pressure of $50 \mathrm{~mm} \mathrm{Hg}$.

Dr Ratcliffe. In the future, if you saw an abnormal SPECT scan, would you alter your technique?

Dr Moraca. You can alter your intraoperative technique to raise your perfusion pressure. However, to my knowledge that has not been shown in many studies to be effective once you get 
beyond a certain pressure, so patients are maintained at that minimal pressure of $50 \mathrm{~mm} \mathrm{Hg}$.

Dr Ratcliffe. Another mechanism of injury might be that the areas of reduced regional cerebral blood flow are more sensitive to embolic injury either because the emboli are preferentially shunted to those areas or because the brain in those areas has less reserve. There is a study by Pollinen that shows that emboli between 160 and $210 \mu \mathrm{m}$ go preferentially to watershed areas for instance.

Another key interesting findings is of silent brain infarcts after CABG. There is a study by Restropo who found that $31 \%$ of CABG patients had abnormal postoperative diffusion-weighted MRI scans. Lesions were small and rounded, and those authors suggested that they were most likely caused by emboli.

Did the patients who had strokes and/or any of the others have postoperative MRI scans? What were the characteristics of the lesions, and specifically were they located in the area of hypoperfusion?

Dr Moraca. There were 3 patients who had perioperative strokes. The first patient had a large left hemispheric stroke and was 1 of our perioperative deaths. The other 2 patients had postoperative strokes. These were in small areas, both on the right and left side. I don't know whether the strokes correlated with the ischemic areas on their SPECTs.

Dr Ratcliffe. What are your plans in the future? Specifically, given the relatively low likelihood of stroke and the benefit or the change that you see with reduced regional blood flow, have you done a power calculation? Do you have an estimate of the number of patients that you would need to study to detect a significant effect on stroke?

Dr Moraca. Determination and measuring of neurologic injury after cardiac surgery can be difficult. Two types of neurologic injury are seen: type 1 , which includes CVAs, both hemorrhagic and ischemic. Type 2 injuries have been described as perioperative cognitive changes. I think what this study really points out is that patients referred for CABG may have a poor physiologic cerebral reserve. They may be unable to tolerate either hypoperfusion or small embolic events, which can occur during cardiac surgery. Thus, one important point of our study is that the etiology of neurologic injury after cardiac surgery may not rest solely on intraoperative events such as clamping techniques, hypoperfusion, inflammation, and embolic events, but may be related to baseline cortical reserve. Ultimately, it may be what the patient brings to the table.

Dr Jay LaBourene (San Francisco, Calif). I was quite stimulated by your study. A couple of questions: Dr Ratcliffe already asked is there correlation of the brain site and I guess you didn't have an answer for that.

With respect to the type 2 injury or perhaps more of the neurocognitive testing, is there any correlation or did you look at that?

Dr Moraca. In this study we did not look at postoperative neurocognitive testing. In our original study in 1998 we were able to demonstrate that poor preoperative SPECT was predictive of neuropsychologic decline. The aim of this study was to define baseline cerebral perfusion in patients referred for CABG.
Dr Labourne. My last question, because I find this so interesting, is there an animal model that might mimic this that could then allow testing to be done with respect to various intraoperative manipulations that may benefit patients who come with a low SPECT score?

Dr Moraca. That's a great question. Unfortunately I don't know if there is an animal model that is available.

J. Scott Rankin (Nashville, Tenn). I apologize for rising again but this is such an exciting study; it's an epiphany really, and correlates so well with our experiences. A few years ago we set out on a program of taking patients at high risk for cerebrovascular injury perioperatively-long-term diabetics, patients with previous strokes, and so on-and doing MRA studies on them. We found that they virtually all had disease, a tremendous amount of small vessel disease in the brain. So we came away from that experience thinking that there was really nothing we could do and the majority of patients had a problem. With the exception of carotid Doppler studies, it wasn't really worth evaluating them, and what we needed to do was to focus on maintaining cerebral perfusion hemodynamically on-pump. So in addition to biocompatible cardiopulmonary bypass systems, good filtering systems, and minimization of pump suckers, we started a program of pulsatile bypass. Theoretically, physiologically pulsatile bypass could maintain flow past an intrinsic obstruction better. We have not scientifically evaluated the data, but over the last several years we have the distinct impression that the stroke rate has fallen. I would like to ask you if you have any experience with pulsatile bypass and what other tricks would you have besides maintaining mean arterial pressure to try to minimize intraoperative stroke rate?

Again, congratulations on a great study.

Dr Moraca. Thank you. I believe that several techniques have been shown in the literature to reduce type 1 neurologic injuries, in particular, single-clamp techniques and pulsatile perfusion. Our institution focuses on short bypass times, the use of aprotinin, single clamp techniques (when feasible), and maintaining optimal perfusion pressures in an attempt to minimize neurologic injury. Pulsatile bypass preserves cerebral autoregulation better in rat models, which is how a lot of these ischemic areas maintain cerebral perfusion. Currently we do not employ pulsatile bypass at our institution.

Dr R. Christopher King (Bremerton, Wash). Is your group aware of any studies where you looked at correlation between your findings with the SPECT scan and intraoperative measuring of cerebral oximetry?

Dr Moraca. I'm not aware of any studies. One interesting aspect about SPECT is you can inject the radioisotope and regional uptake occurs within about 2 minutes without any redistribution for up to 6 hours. Thus, we have employed SPECT to determine cerebral perfusion while patients are on bypass by injecting the radioisotope after instituting bypass, and, once they are out of the operating room, taking them to the radiology suite for a SPECT scan. Amazingly their SPECT uptake will be proportional to their perfusion on bypass. We have noted that patients with poor preoperative cerebral perfusion have markedly impaired perfusion on bypass. 